\title{
PROFILE OF COSMETIC CONSULTATION IN A GENERAL DERMATOLOGY CLINIC
}

\author{
Thapa DP1, Pradhan $S^{2}$
}

${ }^{1}$ Department of Dermatology, Nepal Medical College Teaching Hospital, Attarkhel, Gokarneshwor-8, Kathmandu,

${ }^{2}$ Nursing superviser, Nidan Hospital, Lalitpur, Nepal

\begin{abstract}
Cosmetic Dermatology has garnered tremendous interest all over the world. With the advent of modern technologies and developments, demand for non-surgical aesthetic procedures is on a rise. The purpose of the study is to know the types of cosmetic consultations and procedures being done in a General Dermatology setup. Patients who visited outpatient dermatology department of a multi-speciality, Nidan Hospital, Lalitpur, between May 2017 to April 2018 were included. Details of patients' demographic profile were filled in a preset performa. Other information related to cosmetic consultation including dermatological diagnosis, awareness of cosmetic procedures and cosmetic procedures etc were also included. 160 patients attended the dermatology outpatient during the study period. Seventy four percent of them were females. Thirty six percent of the cases were between 26 years and 35 years. Forty three percent of the cohort were working. Majority of them had Bachelors degree (57\%). Twenty six patients who attended the OPD wanted cosmetic consultation. The type of cosmetic consultation, majority sought for was wrinkles (37\%). Among the cosmetic procedures performed, chemical peeling was done in 59\%, mesotherapy in $16 \%$, mole removal in $12 \%$ and remaining $13 \%$ underwent topical treatment. Statistical significance was seen between Cosmetic consultation and economic status $(\mathrm{p}=0.000)$ and between cosmetic consultation and employment status ( $p=0.007$ ). Almost a quarter of patients attending a general dermatology OPD sought cosmetic consultation. Females, young adults who are employed, well-educated and from good socio-economic status seek cosmetic services.
\end{abstract}

\section{KEYWORDS}

Aesthetic dermatology, cosmetic procedures, chemical peeling

\section{CORRESPONDING AUTHOR}

Dr. Deeptara Pathak

Department of Dermatology,

Nepal Medical College Teaching Hospital,

Attarkhel, Gokarneshwor-8, Kathmandu, Nepal

Email: drdeeptarapathak@yahoo.com

ORCID ID: 0000-0002-1602-415X 


\section{INTRODUCTION}

Dermatology is a speciality which deals with diagnosis, treatment, prevention of skin, skin appendages and mucous membranes. Aesthetic and cosmetic dermatology as a subspecialty of Medical-Surgical Dermatology deals with skin care for healthy skin and enhancing the aesthetic feature of healthy skin as part of beauty and personal image (hygiene, appearance and skin protection). Cosmetic dermatology is a component of dermatology that refers mainly to treatments for healthy skin, and cosmetology is the science and art of caring for and enhancing the aesthetic features of a healthy skin. ${ }^{1,2}$

Dermatologists consultations are usually done for either classic dermatologic disorders or for patients requiring cosmetic dermatology advice and treatment. The patients seeking cosmetic dermatology treatments are driven by the desire to remain eternally young, the importance attached to physical appearance in our society, and the broader concept of health that refers not just to the absence of disorders and diseases, but also to full physical, mental and emotional wellbeing. ${ }^{3}$

The demand for cosmetic dermatology services have increased over recent years manifestly evident by both epidemiological and marketing studies. The sales of cosmeceutical products had grown five-fold from 22 million units to 105 million units between 1973 and 2003 revealing cosmetic dermatology development as per Torras and Lopez et al. ${ }^{4}$

Clinicians must be aware of individuals who usually needs cosmetic procedures to control excessive self and body image concerns that are out of proportion to objective physical findings. An obsession with perfection a term called Body dysmorphic disorder (BDD) is a disorder of selfperception. These individuals are preoccupied with a nonexistent or minimal flaw in appearance. Such patients complain of supposed deformities, ranging from warped eyebrows, oversized nostrils, and tiny genitals to monstrous mouths, making them feel excruciatingly ugly. ${ }^{5}$

This study was undertaken to know the weightage of cosmetic consultation and procedures in a general dermatology setup. Though various procedures like Botulinum toxin injections, chemical peeling, mesotherapy, laser hair removal, hair transplantation etc are carried out but there are no published research data available in this field in the literature from Nepal till date.

\section{MATERIALS AND METHODS}

All patients who visited outpatient dermatology department of Nidan Hospital, Lalitpur between May 2017 to April 2018 were included. All patients who had Dermatology consultation in the outpatient setting were taken informed consent to enrol in the study. Admitted patients seeking dermatology consultation were excluded from the study. Details of patients' demographic profile like age, sex, address, caste, marital status, occupation, economic status, level of education were filled in a preset performa. Among cosmetic consultations, other information including dermatological diagnosis, awareness of cosmetic procedures and cosmetic procedures were also included.

\section{RESULTS}

Of 238 patients availing the dermatology services, 160 patients attending the Dermatology OutPatient Department (OPD) were included in the study. There was female preponderance (74\%) among the patients visiting the OPD. Categorising the patients according to age groups, 36\% were between 26 years to 35 years, $29 \%$ (15 to 25years), 17\% (36 to 45years), $12 \%$ (>45years) and 15\% (<15years). Majority of patients were from Lalitpur (61\%) followed by Kathmandu (30\%), Bhaktapur (5\%) and outside Kathmandu Valley (4\%). Forty nine percent of the patients were married, 35\% unmarried, 3\% separated and $13 \%$ did not specify their marital status. $44 \%$ of patients mentioned their ethnicity as Newari, 29\% Brahmin \& Chetri and 11\% Rai \& Limbu; remaining $16 \%$ were from various minorities group. Majority of the people were employed (43\%), followed by housewives (33\%), students (16\%), retired personnels 5\%; 3\% did not specify their profession. Out of the patients who were employed, 54\% were in teaching profession, $16 \%$ working in private firms, $11 \%$ engineering, $8 \%$ banking, $6 \%$ government jobs, $4 \%$ business and $1 \%$ others. When probed about the Level of education, the majority had Bachelors degree (57\%) followed by higher secondary level (18\%), secondary level (10\%), Masters (11\%), primary level (6\%) and 2\% were Illiterate. Regarding economic status of the patients $75 \%$ belonged to middle class, $21 \%$ higher class and $4 \%$ low socioeconomic status.

$26 \%$ of the patients attending the Dermatology OPD approached for cosmetic consultation and rest had various complaints like acne (18\%), cutaneous infections 18\% (including fungal, bacterial and viral), pigmentary disorders (11\%), 


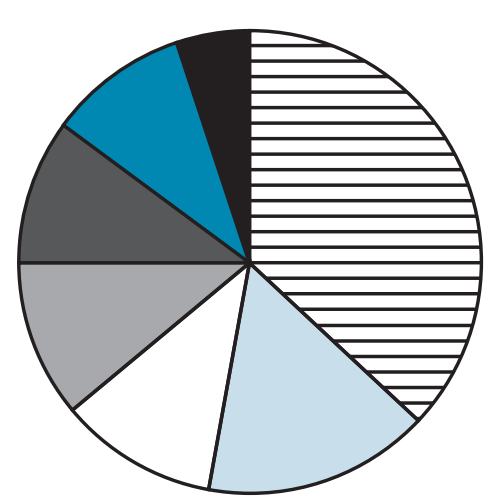

Facial wrinkles

$\square$ Facial rejunivation

Moles removal

$\square$ Hair loss

$\square$ Dilated pores

Hair removal

Fig. 1: Types of Cosmetic Consultation

photo-dermatitis (4\%), eczema and inflammatory disorders (7\%), pruritus (2\%) and others (14\%). Cosmetic consultation were sought for wrinkles (37\%), removal of moles (16\%), dilated pores (11\%), hair removal (11\%), facial rejuvenation (10\%), dull skin (5\%) and hair loss (10\%) (see Fig. 1).

Participants shared that they got to know of cosmetic dermatology either from internet (33\%), or from friends (31\%), radio (19\%), television $(10 \%)$ or newspaper and magazines (7\%). Of all

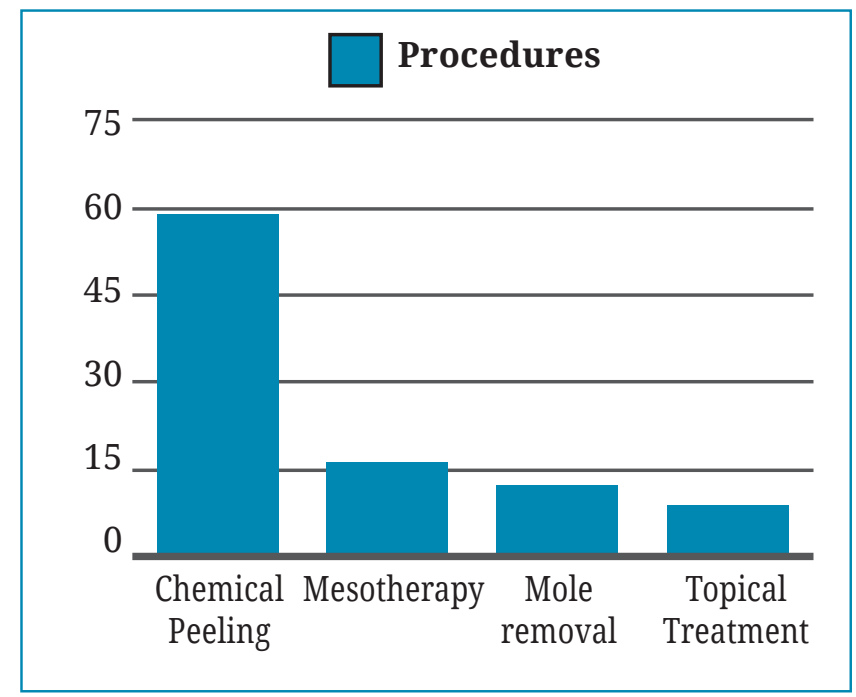

Fig. 2: Procedures done in the Clinic

the procedures done for the patients who sought cosmetic consultation, 59\% underwent chemical peeling, $16 \%$ mesotherapy, $12 \%$ mole removal and remaining $13 \%$ opted for topical treatment (see Fig. 2).

Statistical significant association was seen between cosmetic consultation and economic status $(\mathrm{p}=0.000)$ and also cosmetic consultation and employment status $(\mathrm{p}=0.007)$ but there were no statistical difference seen between cosmetic consultation and sex, age, level of education and places.

\section{Table 1: Aesthetic procedures in percentage country wise in the world}

\begin{tabular}{|lcc|} 
Rank & Country & \% of worldwide Total \\
1 & USA & 21.8 \\
2 & Japan & 11 \\
3 & Brazil & 7.6 \\
4 & Italy & 5.2 \\
5 & Mexico & 4.1 \\
6 & Germany & 3.3 \\
7 & Colombia & 1.4 \\
8 & Thailand & 0.2 \\
9 & Present study & 15 \\
\hline
\end{tabular}

\section{DISCUSSION}

The factors like increase in life expectancy and social expectations to look beautiful have led to increase in general population's interest to extend their youthfulness and to keep a "young and beautiful" body image which will enhance their self-esteem. This has led to rise in the demand of techniques and treatments to improve healthy skins, or when appropriate, to mitigate the evidence of the natural passing of time such as wrinkles, stains, or small residual scars. ${ }^{6}$

Sadick et al found that patients, who had undergone aesthetic treatments, consider health or well-being is connected to mental, emotional, and social consequences associated with their physical appearance. ${ }^{1}$

Generally, there are significantly positive changes in the social life of patients. A persons self-esteem and behaviour toward others, is influenced by their physical attraction and it is directly related to self-confidence and social acceptance..$^{1,7}$ Information regarding improving self-appearance is in great demand these days and for which Dermatologist consultation are sought for by the patients. ${ }^{8}$ According to Pierre, desire to remain beautiful is a result of pride, pretentiousness a desire to get admiration and to look better than anyone else. ${ }^{4}$ Beauty has become a culture and human beings deny to accept their fate but seek to improve it hence if medicine has given years to life then aesthetic medicine and surgery have gifted life to years. ${ }^{9}$ In this era, due to rise in modern technologies and improved 
practices, have incredibly boosted the rise of cosmetic procedures as per the statistics from West, with trends more for non-surgical than surgical procedures. In last decade the demand for botulinum toxin injection, hyaluronic acid fillers, laser hair removal, microdermabrasion and chemical peels have been acclaimed as the top non-surgical procedures. There are very limited data of such procedures from Nepal. However due to demand wide range of cosmetic procedures is being practiced these days. Various dermato-surgical, laser and non-laser skin rejuvenation procedures, acne scar treatment, anti-wrinkle treatment, pigmentation, tattoo removal, stretch mark removal, hair restoration, hair removal, phototherapy and mesotherapy are some of the prevailing aesthetic procedures being practiced in Nepal. ${ }^{10}$ In our setup, about $26 \%$ of patients came to seek cosmetic consultation .

According to International study on Aesthetic/ Cosmetic procedures (ISAPS) worldwide in 2017 the 5 topmost non surgical procedures were Botulinum toxin injections (83\%), fillers (78\%), chemical peel (31\%), Hair removal (21\%), non surgical skin tightening (21\%). The total non surgical procedures worldwide according to countries as shown in Table $1 .{ }^{11}$ Statistical analysis from the American Society for Aesthetic Plastic Surgery (ASAPS) in 2005, found nonsurgical procedures making up $81 \%$ of the total. ${ }^{12}$ Statistical data from the ASAPS in 2017 showed the top most 5 non surgical procedures being Botulinum toxin injections, fillers, non surgical fat reduction, hair removal and Chemical peels. According to the percentage change as per ASAPS in 5 years (20122017) there is increase demand in non surgical fat reduction about (217.3\%), fillers (85\%), Botulinum toxins injections about (30\%) and chemical peels (26.1\%), but demand has been seen to decrease for hair removal (laser or pulse light) compared to 2012 year statistics. In our set up the most common procedures done was chemical peeling as seen in 59\%. ${ }^{13}$ As per ISAPS statistics(2017), country wise percentage distribution of chemical peeling was seen highest in Italy (65\% ) followed by USA $41 \%$, Brazil (29\%), Germany (21\% )and least in Thailand (2\%). Though we have also found chemical peeling been done in 59\% cases but we don't have studies to compare within our country, as aesthetic dermatology in this part of the world is emerging.

There was statistical significance between cosmetic consultation and economic status; people belonging to middle class and high socio-economic status opt more for aesthetic procedures, which holds true as these cosmetic procedures are costly.

For the major source of public information regarding cosmetic dermatology, Doheyan et al found $93.8 \%$ people had received their information from television, Adejeji et al found $49.8 \%$ from television and Ahmed et al found $88 \%$ from internet which is similar to our study (33\%), while in a study from Nepal among health workers, $34 \%$ got to know about cosmetic dermatology from medical textbooks. ${ }^{14-17}$ There were also significant various factors attributing for increasing demand for aesthetic dermatological procedures like secular consumer culture, economic abundance, medical advances, media driven hype and lack of regulatory control. Increased demand for aesthetic procedures has resulted in an ever-increasing number of beauty parlour and charlatans providing scientifically unproven aesthetic services and making unproven claims. ${ }^{9,18}$

At present in many countries as well as in Nepal there is no proper accreditation process to regulate the practice of aesthetic medicine. The practice of aesthetic medicine should not be exempted from the need for structured training and accreditation which ultimately serves to protect the public from unproven and unsafe treatments. ${ }^{18}$

In Nepal even though cosmetic dermatology is in infancy, we do find people seeking aesthetic consultation. Mostly young educated and working female with good socio-economic background seek for the services. Hence we need to develop this specialty in dermatology as a structured and well regulated training program.

\section{REFERENCES}

1. Sadick NS. The impact of cosmetic interventions on quality of life. Dermatol Online J 2008; 14: 2.

2. Elisman AB. Needs and Demands in Cosmetic Dermatology. Actas Dermosifiliogr 2009; 100: 87-8.

3. Torras H, López X. Situación de la cosmetología y de la estética dentro de la dermatología. Evolución de la dermocosmética en los últimos 30 años. Med Cutan Iber Lat Am 2005; 33: 1-5.
4. Vashi NA.Obsession with perfection: body dysmorphia. Clin Dermatol 2016; 3: 788-91

5. Martinez Gonzalez MC, Martinez Gonzalez RA, Guerra Tapia A. Aesthetic dermatology and emotional well-being questionnaire. J Cosmet Dermatol 2014; 13: 336-45

6. Von Soest T, Kvalem IL, Skolleborg KC et al. Psychosocial changes after cosmetic surgery: a 
5-year follow-up study. Plast Reconstr Surg 2011; 128: 765-72 .

7. Matarasso SL. Introduction to cosmetic surgery. Semin Dermatol 1994; 13: 60-3.

8. Pierre FF. The Lorenz theory of Beauty. J Cosmet Dermatol 2002; 1: 131-136.

9. Goh $\mathrm{Cl}$. The need for Evidence based Aesthetic Dermatology Practice. J Cuban Aesthet Surg; 2009; 2: 65-71.

10. Karn D. Aesthetic Practice in Nepal. Kathmandu Univ Med J 2013;11:109

11. ISAPS Global statistics 2017.Available from: http:// www.isaps.org> medical professionals

12. American Society of Aesthetic Plastic Surgery. 11.5 million cosmetic procedures in 2005. [published on 2006 Feb]. Available from: http://www.surgery. org/media/news-releases/115-million-cosmeticprocedures-in-2005.

13. Statistics American Society for Aesthetic Plastic Surgery 2017. Available from: https://www.surgery. org/media/statistics
14. Adedeji OA, Oseni GO, Olaitan PB. Awareness and attitude of healthcare workers to cosmetic surgery in osogbo, Nigeria. Surg Res Pract 2014; 2014: 869567.

15. Doheyan AI, Saad AA, Haidar AA, Fwzan HA, Askar JA, Malki FA et al. Attitude and Practices Concerning Cosmetic Surgery among Female Medical Students at the university Hospital, King Said University, Riyadh,Saudi Arabia. BJMMR 2016; 14: 1-10.

16. Ahmed M, Mohammad H, Ahmed N. Views of College students on plastic surgery. World J Plast Surg 2013; 2:104-10.

17. Thapa DP. Awareness of Cosmetic Dermatology procedures among health workers in a tertiary care Hospital. Indian Dermatol Online J 2019; 10: $139-43$

18. Tan KB. Aesthetic medicine: a health regulator's perspective. Clin Governance 2007; 12: 13-25. 